\title{
A Clinical-Radiomics Nomogram Based on Computed Tomography for Predicting Risk of Local Recurrence After Radiotherapy in Nasopharyngeal Carcinoma
}

\author{
Chaohua Zhu ${ }^{1 \dagger}$, Huixian Huang ${ }^{1 \dagger}$, Xu Liu ${ }^{1 \dagger}$, Hao Chen ${ }^{1 \dagger}$, Hailan Jiang ${ }^{1}$, Chaolong Liao ${ }^{1}$, \\ Qiang Pang ${ }^{1}$, Junming Dang ${ }^{2}$, Pei Liu ${ }^{3}$ and Heming Lu ${ }^{1 *}$
}

'Department of Radiation Oncology, People's Hospital of Guangxi Zhuang Autonomous Region, Nanning, China, ${ }^{2}$ Department of Clinical Oncology, Guangxi University of Chinese Medicine, Nanning, China, ${ }^{3}$ Department of Clinical Oncology, Youjiang Medical University of Nationalities, Baise, China

OPEN ACCESS

Edited by:

Po-Hsiang Tsui,

Chang Gung University, Taiwan

Reviewed by:

Chuanben Chen,

Fujian Provincial Cancer Hospital, China

Fei Han,

Sun Yat-sen University Cancer Center

(SYSUCC), China

*Correspondence:

Heming LU

luhming3632@163.com

tThese authors have contributed equally to this work

Specialty section:

This article was submitted to Cancer Imaging and Image-directed Interventions,

a section of the journa

Frontiers in Oncology

Received: 04 December 2020 Accepted: 22 February 2021

Published: 18 March 2021

Citation:

Zhu C, Huang H, Liu X, Chen $H$, Jiang $H$, Liao C, Pang Q, Dang J, Liu P and Lu H (2021) A Clinical-Radiomics Nomogram Based on Computed Tomography for Predicting Risk of Local Recurrence After Radiotherapy in Nasopharyngeal Carcinoma. Front. Oncol. 11:637687.

doi: 10.3389/fonc.2021.637687
Purpose: We aimed to establish a nomogram model based on computed tomography (CT) imaging radiomic signature and clinical factors to predict the risk of local recurrence in nasopharyngeal carcinoma (NPC) after intensity-modulated radiotherapy (IMRT).

Methods: This was a retrospective study consisting of 156 NPC patients treated with IMRT. Radiomics features were extracted from the gross tumor volume for nasopharynx (GTVnx) in pretreatment CT images for patients with or without local recurrence. Discriminative radiomics features were selected after $t$-test and the least absolute shrinkage and selection operator (LASSO) analysis. The most stable model was obtained to generate radiomics signature (Rad_Score) by using machine learning models including Logistic Regression, K-Nearest neighbor, Naive Bayes, Decision Tree, Stochastic Gradient Descent, Gradient Booting Tree and Linear Support Vector Classification. A nomogram for local recurrence was established based on Rad_Score and clinical factors. The predictive performance of nomogram was evaluated by discrimination ability and calibration ability. Decision Curve Analysis (DCA) was used to evaluate the clinical benefits of the multi-factor nomogram in predicting local recurrence after IMRT.

Results: Local recurrence occurred in 42 patients. A total of 1,452 radiomics features were initially extracted and seven stable features finally selected after LASSO analysis were used for machine learning algorithm modeling to generate Rad_Score. The nomogram showed that the greater Rad_Score was associated with the higher risk of local recurrence. The concordance index, specificity and sensitivity in the training cohort were 0.931 (95\%Cl:0.8765-0.9856), 91.2 and 82.8\%, respectively; whereas, in the validation cohort, they were 0.799 (95\%Cl: 0.6458-0.9515), 79.4, and $69.2 \%$, respectively.

Conclusion: The nomogram based on radiomics signature and clinical factors can predict the risk of local recurrence after IMRT in patients with NPC and provide evidence for early clinical intervention.

Keywords: nasopharyngeal carcinoma, radiomics, prognostic prediction, nomogram, local recurrence 


\section{INTRODUCTION}

Nasopharyngeal carcinoma (NPC) prevails in Southern China. Intensity-modulated radiation therapy (IMRT) is the primary treatment modality for NPC patients and the 5 -year survival rate has reached over $80 \%$ (1). However, it has been reported that about $10 \%$ of patients will eventually experience local recurrence within 3 years after IMRT (2). Therefore, accurate prediction of the risk of local recurrence is crucial to initiate an early individualized intervention (3).

To date, clinical characteristics such as tumor-node-metastasis (TNM) staging, Epstein-Barr virus (EBV) DNA level and tumor volume, are the main prognostic factors for patients with NPC. It has been reported that different responses and survival outcomes may occur after IMRT in patients with the same clinical stage. TNM stage based on anatomy is not always a reliable prognostic factor to precisely predict the recurrence (4). Therefore, it is necessary to find more reliable and practical markers that can reveal tumor heterogeneity before treatment. In recent years, some studies have shown that a large number of molecular markers are associated with tumor growth, metastasis and prognosis in NPC patients $(5,6)$.It has been acknowledged that with the advances in genomics, genes that drive oncogenesis and disease progression are composed of different genotype subsets. The spatial heterogeneity of solid tumors cannot be revealed by a single invasive biopsy. It has been confirmed that image-based phenotypes can be used to quantify the spatial heterogeneity of tumors (7). Recently, some studies have indicated that radiomics signature generated by using radiomics features which are extracted from high-quality imaging data might be served as imaging markers to predict treatment outcome (8-11).

Radiomics is produced by big data and medical image-assisted diagnosis technology. Radiomics features extracted from region of interest (ROI) in medical images by using a large number of automatic and semi-automatic algorithms can describe in-depth information, such as tumor phenotype and heterogeneity (12, 13). Precise radiotherapy cannot be achieved without medical imaging. CT images contain tumor morphology, texture, and gray level information, which play an important role in target delineation, planning design and dose calculation in radiotherapy for NPC.

Rad_Score is one of the biological markers which reflect the spatial heterogeneity of tumors. In the present study, through data mining method, Rad_Score was generated from radiomics features which were extracted from the gross tumor volume for nasopharynx (GTVnx) in planning CT. We expected that Rad_Score combined with potential clinical factors may help to build and validate a nomogram model for risk prediction of local recurrence in NPC patients.

\section{MATERIALS AND METHODS}

\section{Patients}

A retrospective analysis was performed for NPC patients who were treated with IMRT in the People's Hospital of Guangxi Zhuang Autonomous Region between January 2016 and December 2017. Eligibility criteria were as the following: histologically proven undifferentiated, non-keratinized carcinoma; after definitive IMRT; stages T1-T4 according to the 2010 AJCC Staging System. Exclusion criteria were as the following: incomplete CT images; lost to follow-up; regional recurrence or distant metastasis after IMRT; death not caused by local recurrence.

\section{CT Scan}

CT images were acquired from a simulation CT scanner (Somatom Sensation Open, Siemens Medical Solutions, Erlangen, Germany) for all patients. Scanning parameters were as follows: $120 \mathrm{kVp}, 250 \mathrm{mAs}$, FOV $500^{*} 500 \mathrm{~mm}, 2 \mathrm{~mm}$ slice. Fifty-five seconds before CT scan, patients were injected with $95 \mathrm{ml}$ iodixanol. CT image reconstruction was based on filtered back projection (FBP) algorithm.

\section{Target Delineation and Treatment}

The target delineation was in accordance with the International Commission on Radiation Units and Measurements Reports 50 and 62. GTVnx included gross disease determined by CT, MRI or PET-CT. Planning target volume for nasopharnx (PTVnx) was generated by adding 3-mm margin to GTVnx. The plans were designed and optimized using the Pinnacle inverse planning system. The prescribed radiation dose was 70Gy at 2.12 Gy-2.3 Gy per fraction, delivered to PTVnx. All patients were treated once daily, five fractions weekly. Image-guided cone beam computed tomography (СBCT) was performed in all patients before each treatment session.

\section{CT Radiomics Signature}

The radiomics workflow is shown in Figure 1: (1) image acquisition and segmentation of ROI; (2) Feature extraction; (3) Feature dimension reduction and Rad_Score generation; (4) Establishment and validation of Nomogram model.

The GTVnx was defined as ROI. Radiomics features were extracted from ROI in IBEX (Imaging Biomarker Explorer, Version 1.0) (14). Radiomics features included Shape, Gray Level Co-occurrence Matrix 25, Gray Level Co-occurrence Matrix 3, Gray Level Run Length Matrix 25, Intensity Direct, Intensity Histogram, Neighbor Intensity 25, Neighbor Intensity Difference 3 and Intensity Histogram Gauss Fit.

The radiomics features extracted from local recurrent and non-recurrent patients were first tested by analysis of variance (ANOVA). The least absolute shrinkage and selection operator (LASSO) algorithm was adopted in selecting the most significant features and feature dimension reduction. The coefficient value of insignificance features was reduced to 0 using 10-fold cross verification.

The selected radiomics features were put into seven types of machine learning classifiers for modeling: K-nearest Neighbor (KNN), Logistic Regression (Log), Naive Bayes (NB), Decision Tree (DT), Stochastic Gradient Descent (SGD), Gradient Boosting Trees (GBT), and Linear Support Vector Classification (SVC).The performance of the predictive models was evaluated by analysis of the area under the curve (AUC) of the receiver operating characteristic (ROC). The most stable classification model was obtained referring to the recall rate and the score of 


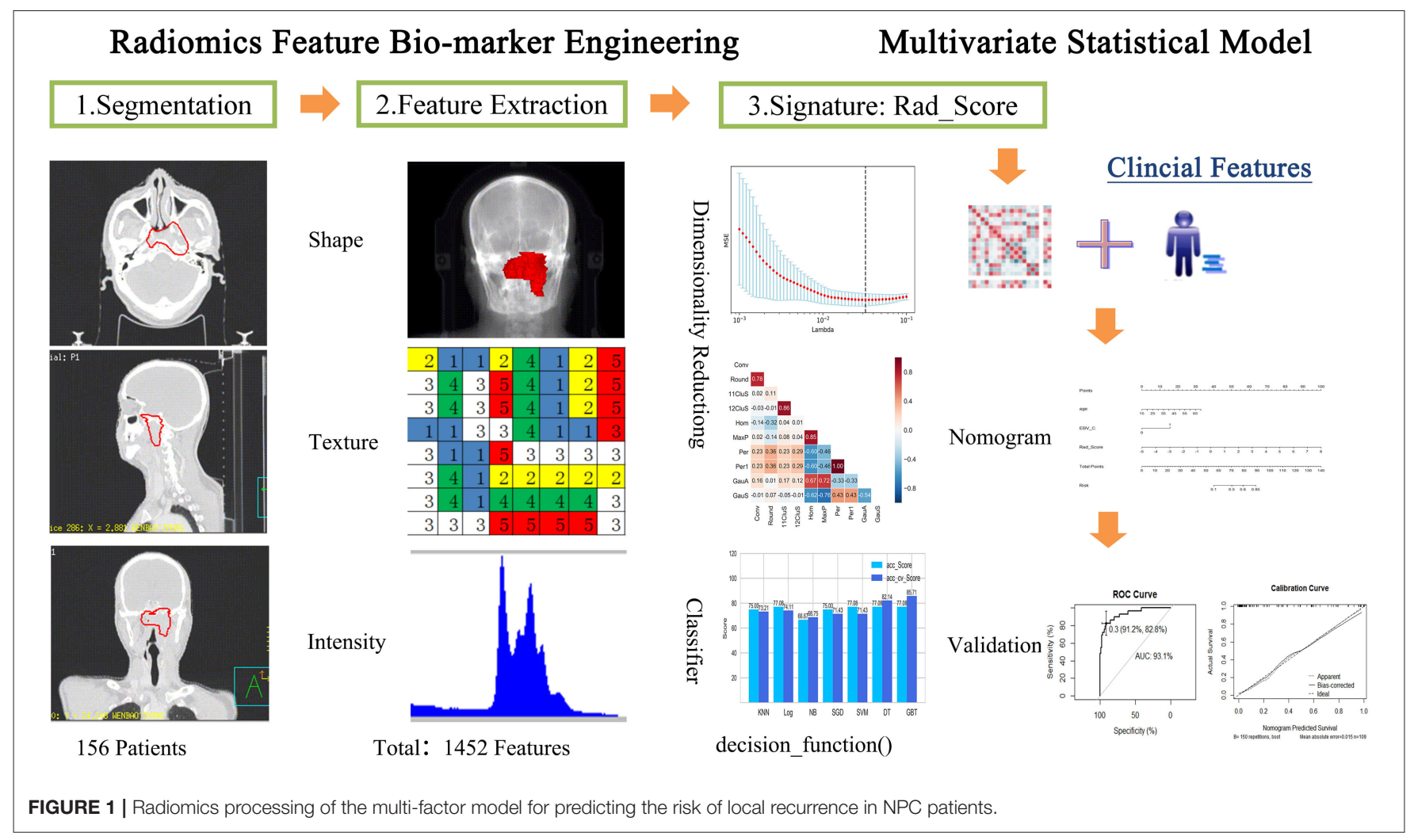

cross-validation. Finally, Rad_Score was generated according to the optimal classification model.

A nomogram was established based on Rad_Score and clinical characteristics including age, gender, T-stage, and changes of EBV-DNA levels in plasma after treatment using logistic regression analysis. Change in EBV-DNA level relative to pretreatment baseline was considered as a dummy variable and denoted as EBV_C: 1 if EBV-DNA copies remained high or increased compared to its pre-treatment level, otherwise it was 0 .

Stepwise method was used to perform variable filtering for simplifying the prediction model, and the optimal nomogram was finally obtained. Predictive performance of the nomogram was evaluated by discrimination and calibration in the training cohort and in the validation cohort. Discrimination was defined as consistency between the predictive ability and the actual recurrence after IMRT based on The Harrell's Concordance Index (C-index). Calibration was defined as consistency between the probability of the outcome predicted by the nomogram and the actual probability. Decision Curve Analysis (DCA) was used to evaluate the clinical benefits of multi-factor nomogram in predicting local recurrence after IMRT.

\section{Statistical Analyses}

The chi-square test for radiomics features was performed using the python 3.7 software package SciPy. Feature dimension reduction and feature modeling were established by LASSO algorithm using the Sklearn package. Multi-factor nomogram was analyzed using the "RMS" and "RMDA" package running in $\mathrm{R}$ software, version 4.0.2. For all statistical tests, a probability value (p) of $<0.05$ was considered statistically significant.

\section{RESULTS}

\section{Patient Characteristics}

One hundred and fifty-six patients were enrolled into either the training cohort or the validation cohort in a ratio of $\sim 2: 1$ (109:47). There were no significant differences in age, gender, Tstage, or EBV_C between the training cohort and the validation cohort. The patient characteristics included age, gender, T-stage, and plasma EBV_C. The patient characteristics are listed in Table 1.

\section{Feature Extraction and Selection}

A total of 1,452 radiomics features were initially extracted from ROI in IBEX and 1,027 features with significant differences between local recurrence and non-recurrence patients were finally selected using ANOVA. The loss function reached the minimum value when Lambda is 0.032375 adopting LASSO dimension reduction $(15,16)$ (Figure 2).

Ten radiomics features were selected and classified as follows: Shape (Convex, Conv; Roundness, Round), GrayLevelCooccurenceMatrix (11-7ClusterShade, 11CluS; 12-7ClusterShade, 12CluS; 7-4 Homogeneity2, Hom; 64MaxProbability, MaxP), IntensityDirect (65Percentile, 
Per; 65Percentile1, Per1) and IntensityHistogramGaussFit (1GaussAmplitude, GaussA; 1 GaussStd, GaussS). The correlation test of 10 features is shown in Figure 3. One radiomics feature was selected from those with a correlation coefficient $>0.8$, and finally, seven stable selected features (Conv, Round, 11CluS, Hom, Per, GaussA, GaussS) were used for machine learning algorithm modeling (Figure 3).

The performance comparison of seven machine learning classifiers for modeling on the training cohort is shown in Figure 4. The GBT model got the highest cross-validation score of 85.71, with an AUC of the ROC curve was 0.9 in the validation cohort. Finally, Rad_Score which was defined as the distance from the coordinate point composed of radiomics features to the separating hyperplane was generated according to the GBT model. The training cohort and validation cohort met the requirement of independence as

\begin{tabular}{lccc}
\hline \multicolumn{2}{l}{ TABLE 1 | Patient characteristics. } & & \\
\hline Characteristics & Training cohort & Validation cohort & $\boldsymbol{p}$ \\
\hline Age (y, mean \pm SD) & $46.49 \pm 10.62$ & $48.38 \pm 10.21$ & 0.165 \\
Sex & & & 0.423 \\
Female & 39 & 13 & \\
Male & 70 & 34 & 0.566 \\
T stage & & & \\
T1 & 9 & 5 & \\
T2 & 30 & 14 & \\
T3 & 45 & 14 & \\
T4 & 25 & 14 & \\
EBV_C & & & \\
1 & 89 & 39 & \\
0 & 20 & 8 & \\
Rad_Score, median & $-0.39(-4.75$ to 7.53$)$ & $-0.61(-3.73$ to 4.58$)$ & \\
(interquartile range) & & & \\
\hline
\end{tabular}

the $P$-value of the chi-square test for Rad_Score was $>0.05$ (Table 1).

\section{Nomogram Establishment}

A nomogram of local recurrence was finally obtained based on Rad_Score and clinical factors, including age, gender, T-stage, and EBV_C using logistic regression analysis. The differences in age, EBV_C and Rad_Score were statistically significant according to the correlation analysis of the selected variables (Table 2). The actual scores with different variables corresponded to the above point coordinate. Age and Rad_Score were read out with specific values. It got 15 score if the plasma EBV-DNA level increased after IMRT. The sum of the scores of all variables was the total points, and the corresponding risk graph could predict the risk of local recurrence in patients with NPC (Figure 5).

\section{Nomogram Validation}

The concordance index, specificity and sensitivity in the training cohort were 0.931 (95\%CI: 0.8765-0.9856), 91.2 and $82.8 \%$, respectively; whereas, in the validation cohort, they were 0.799 (95\%CI: 0.6458-0.9515), 79.4 and 69.2\%, respectively (Figures 6A,B).

The calibration curves in the training cohort and the validation cohort are shown in Figures 6C,D. The prediction model showed that the probability distribution of the validation cohort was similar to that of the training cohort. In the validation cohort, when the predicted probability was $<0.3$, the risk of recurrence was underestimated, and when the predicted probability was $>0.3$, the risk of recurrence was overestimated.

\section{Clinical Benefits of Nomogram}

In the training cohort, DCA was used to evaluate the differences in clinical benefits of Rad_Score, clinical factors and nomogram of predicting local recurrence in NPC patients. The DCA curve showed that Rad_Score and Nomogram were better predictors for local recurrence at any given threshold probability than clinical characteristics alone (Figure 7).
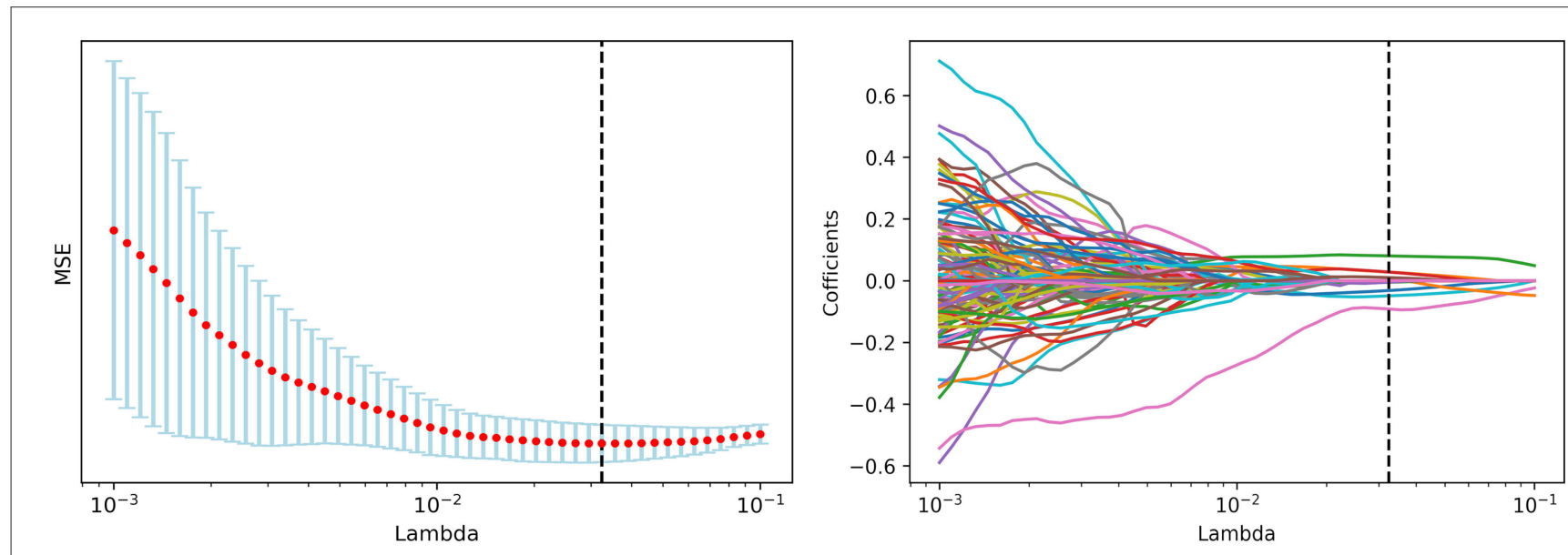

FIGURE 2 | LASSO dimension reduction and Lambda value. 

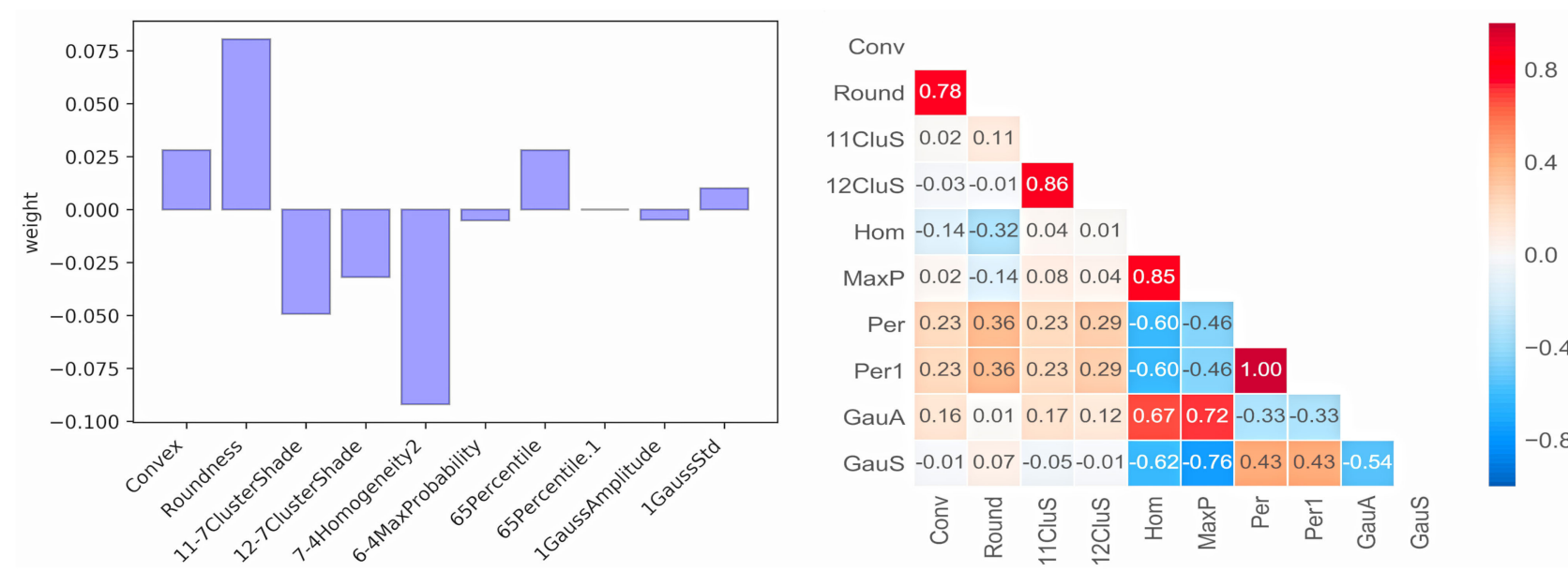

FIGURE 3 | Weight and correlation test of radiomics features.

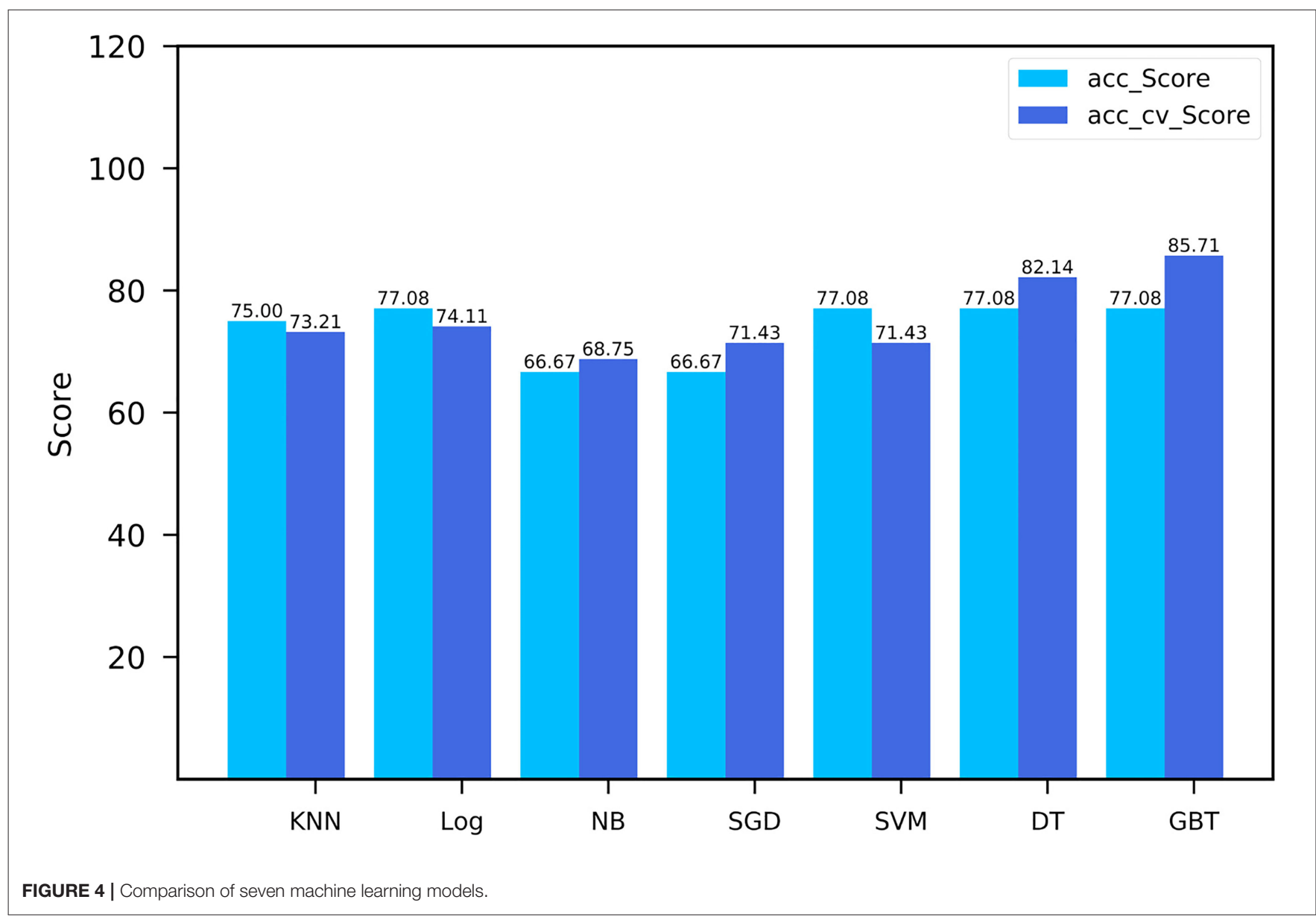

\section{DISCUSSION}

It has been one of the major interests for clinicians to provide evidence for early intervention based on the establishment of prognostic prediction model for patients with NPC. Clinical factors selected for predicting outcome vary with time periods and research fields. In this study, Rad_Score was calculated using radiomics signatures extracted from GTVnx of CT images in 
NPC patients. A multi-factor logistic regression prediction model was built based on clinical factors and Rad_Score to evaluate the risk of local recurrence after IMRT.

Vallières et al. (17) extracted 1,615 radiomics features (quantifying tumor image intensity, shape and texture) and established a prognostic prediction model to evaluate local recurrence and distant metastasis by performing analysis of FDG-PET and CT images in 300 patients with head-and-neck cancer. The survival analysis found that the model was specific for predicting tumor recurrence and metastasis (local recurrence: $\mathrm{AUC}=0.69$; distant metastasis: $\mathrm{AUC}=0.86$ ). Zhang et al. (18) explored the potential of radiomics features based on MRI images in predicting disease progression in patients with advanced NPC.

TABLE 2 | Multivariable logistic regression.

\begin{tabular}{lllll}
\hline Variable & Estimate & Std. Error & z-value & $\boldsymbol{P}$ \\
\hline Intercept & -8.17 & 2.457 & -3.325 & $0.0008^{*}$ \\
Gender & 0.801 & 0.704 & 1.138 & 0.255 \\
Age & 0.089 & 0.039 & 2.273 & $0.023^{*}$ \\
T_stage & 0.514 & 0.413 & 1.243 & 0.213 \\
EBV_C & 2.529 & 0.816 & 3.099 & $0.0019^{*}$ \\
Rad_Score & 1.148 & 0.306 & 3.747 & $0.000179^{*}$ \\
\hline
\end{tabular}

${ }^{\star} P<0.05$.
In this study, 970 initial features were extracted from MRI images of 118 patients, and eight features were found to be related to disease progression through dimension reduction analysis. In the present study, we acquired seven radiomics features from GTVnx on simulation CT after two-step dimension reduction. These radiomics features can describe tumor shape, texture, histogram intensity. In order to obtain Rad_Score with strong generalization ability, it is necessary to select a more appropriate machine learning model for specific conditions. Different from the commonly used LASSO, we put the selected radiomic features into seven types of machine learning classifiers for modeling and calculated the Rad_Score of each patient with the most stable classification model.

It is recognized that MRI have high resolution for soft tissue, and fusion of MRI and CT images are often recommended for the delineation of tumor target in radiotherapy. In the present study, we also fused MRI with CT images to delineate the tumor target. Thus, the acquired radiomic features (Convex and Roundness) also contained information about the shape features in MRI images. Zhang et al. (19) established nomogram of local recurrence risk prediction using MRI images and clinical characteristics (gender, age, hemoglobin, N-stage) before treatment. The C-index of the model was 0.74 (95\%CI: $0.58-$ 0.85). This was consistent with our findings. The C-index of the validation cohort in our study was 0.799 (95\%CI: 0.646-0.952). The possible reasons for the slight difference are

\section{Points}

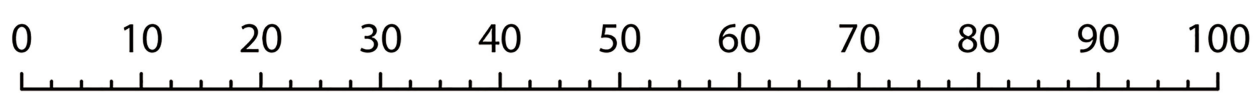

\section{age}

EBV_C

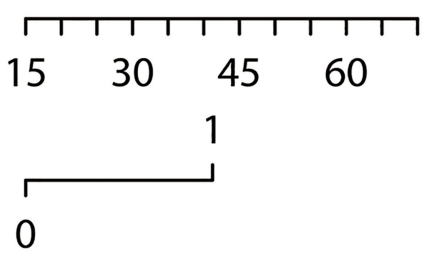

\section{Rad_Score}

\begin{tabular}{llllllllllllllll}
\hline-5 & -4 & -3 & -2 & -1 & 0 & 1 & 2 & 3 & 4 & 5 & 6 & 7 & 8
\end{tabular}

\section{Total Points}

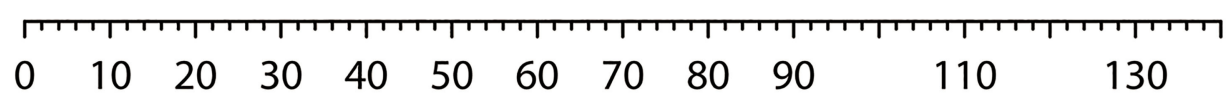

\section{Risk}

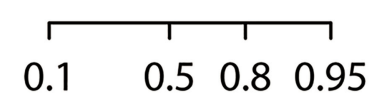

FIGURE 5 | Nomogram for risk prediction of local recurrence in NPC patients after IMRT; Features contained in the training cohort are age, EBV_C and Rad_Score. 


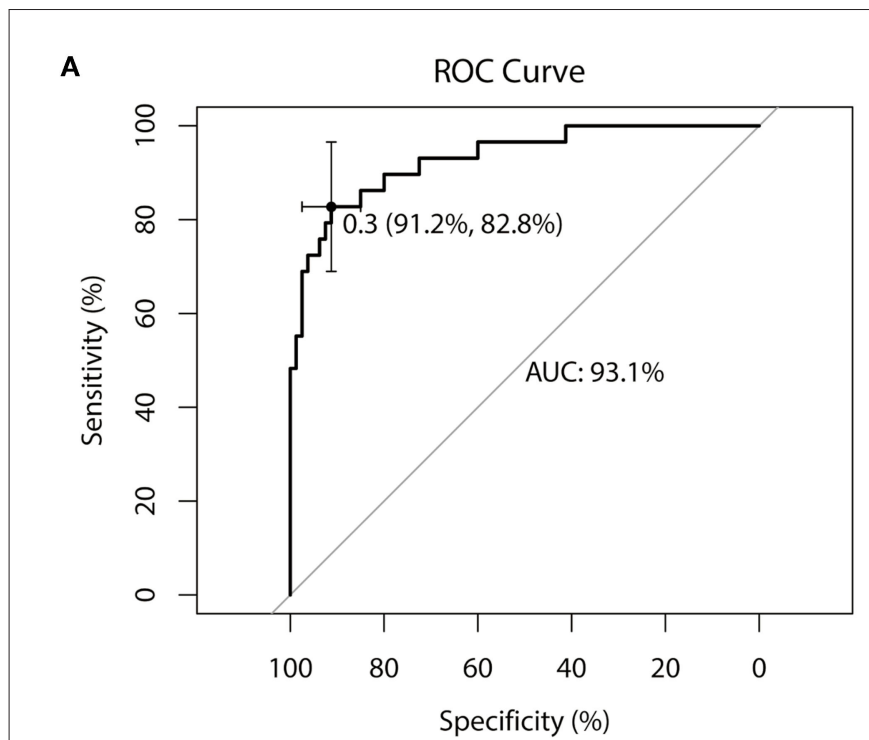

C

\section{Calibration Curve}

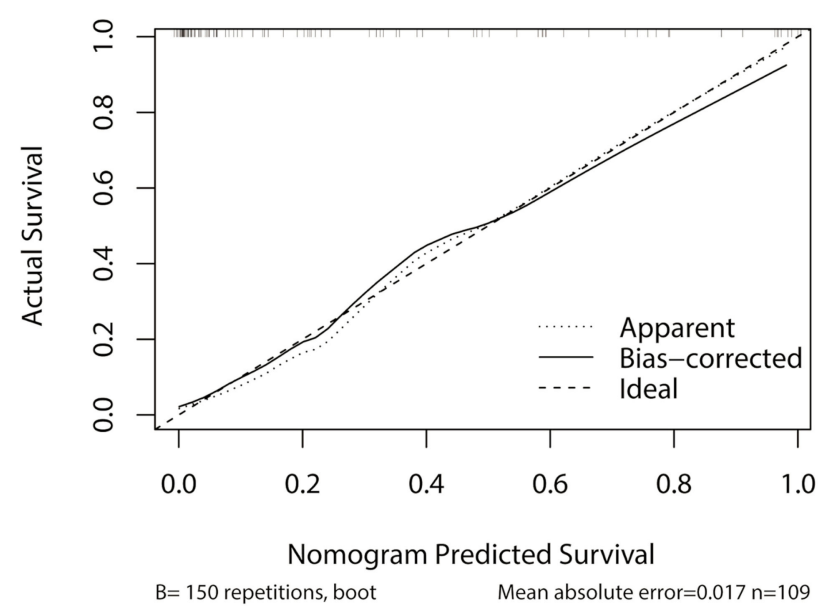

B

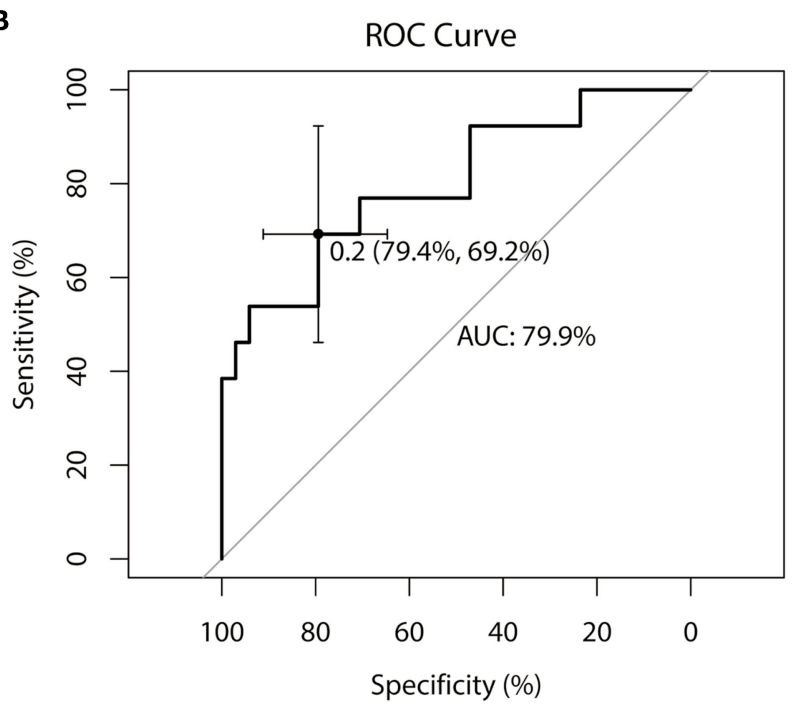

D

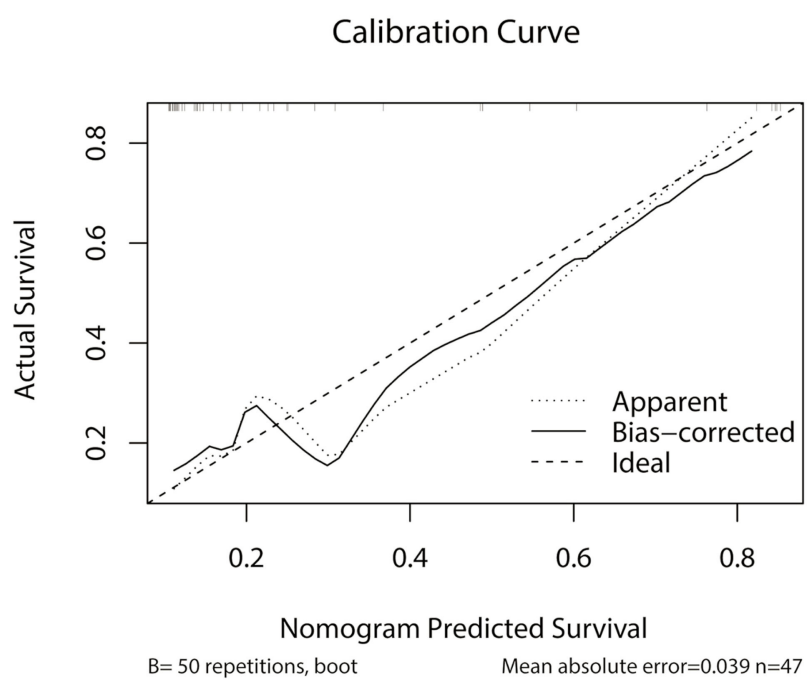

FIGURE 6 | Nomogram validation: (A) discrimination curve in the training cohort; (B) discrimination curve in the validation cohort; (C) calibration curve in the training cohort; (D) calibration curve in the training cohort.

due to different image machines, different type of machine learning classifiers for modeling and different selection of clinical characteristics.

There have been a number of studies on multi-factor analysis of clinical factors to predict prognosis of NPC. Tang et al. (20) established a nomogram for predicting disease-free survival based on age, gender, body mass index (BMI), T-stage, $\mathrm{N}$ stage, C-reactive protein, Lactate Dehydrogenase (LDH), and plasma EBV-DNA levels. Sun et al. (21) used age, N-stage, number of lesions, site of metastasis and plasma EBV-DNA level to establish a nomogram predicting metastasis and survival. It has been reported that plasma EBV-DNA level was an important biomarker for predicting the prognosis of NPC patients $(22,23)$. Plasma EBV-DNA level remained low or negative in clinical remission condition after treatment, but would rise again when recurrence or metastasis occurred. $\mathrm{Li}$ et al. (24) found that the plasma EBV-DNA level in local recurrent NPC patients was positive by $51-67 \%$. Huang showed that age was an important factor for predicting prognosis of NPC (25). In the present study, the clinical factors included age, gender, T-stage, and plasma EBV-DNA levels. Similar to the above-mentioned results, we found that age and plasma EBV_C were significantly correlated with local recurrence. However, there was no significant correlation between T-stage and local recurrence. Jiang et al. (26) reported that, although NPC patients with T4 stage may have a high recurrence rate, there was no difference in the local control rate. 


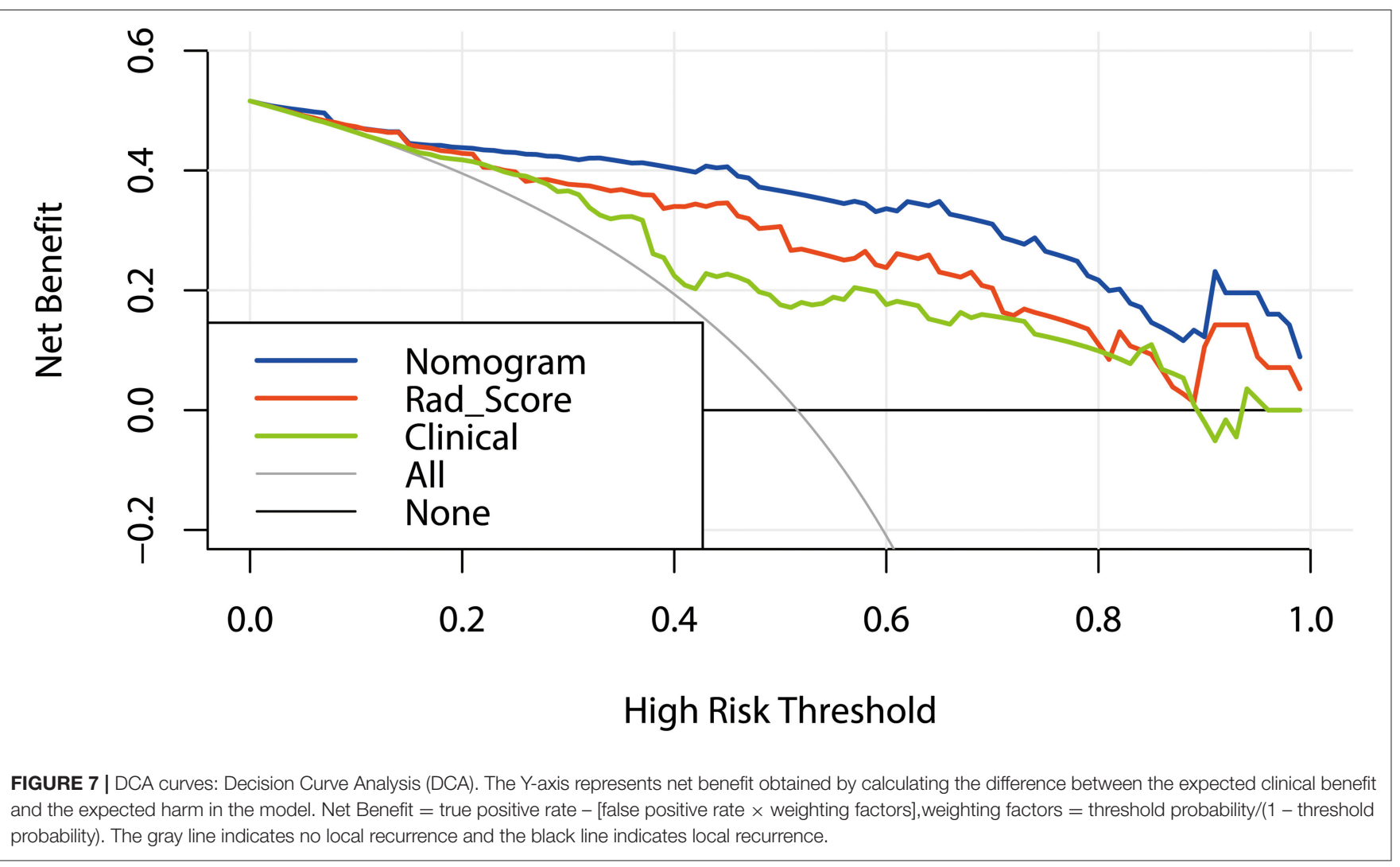

Nomogram has been widely used as predictive models in studies about cancer prognosis (27). It can comprehensively analyze the predictive ability of various factors to meet the modeling requirements based on biomarkers and clinical characteristics, so as to achieve personalized therapy. In this study, nomogram established by Rad_Score and some clinical factors could well-describe the heterogeneity of NPC. It could eliminate the need for re-delineation of GTVnx and avoid interobserver errors because the radiomics features were extracted from the radiotherapy planning. In addition, the shape features of GTVnx contained MRI image information. The validated nomogram found that both the training cohort and the validation cohort had a high degree of discrimination. In the validation cohort, there was a difference in the prediction probability in the calibration, which was possibly attributed to the small sample size and the small positive sample size. The DCA curve showed the comparison of clinical benefit in predicting the risk of local recurrence between clinical characteristics, Rad_Score and nomogram, and the nomogram was found to have better predictive performance than the other two. However, it should be noted that nomogram's predictive performance varies with different clinical treatment models in practical applications.

Our study had some limitations. First, the extracted radiomics features were mainly texture and histogram features of CT and shape features of MRI. The spatial heterogeneity of tumors may be more precisely presented if functional images like functional magnetic resonance image (fMRI) or PET are integrated. Second, the predictive model might perform better if current radiomics which was based solely on spatial heterogeneity of solid tumors is combined with genomic. Finally, independent sample test was conducted in a single institution in this study. Multi-center, large-sample size studies are needed to establish nomogram in more comprehensive clinical settings.

\section{CONCLUSION}

In conclusion, the nomogram based on CT radiomics signature can predict the risk of local recurrence after IMRT in patients with NPC and provide evidence for early clinical intervention.

\section{DATA AVAILABILITY STATEMENT}

The raw data supporting the conclusions of this article will be made available by the authors, without undue reservation.

\section{ETHICS STATEMENT}

The studies involving human participants were reviewed and approved by the Institutional Review 
Board of the People's Hospital of Guangxi Zhuang Autonomous Region. The patients/participants provided their written informed consent to participate in this study.

\section{AUTHOR CONTRIBUTIONS}

$\mathrm{CZ}$ conceived the project and performed the experiment. $\mathrm{HH}$ wrote the paper. XL, HC, HJ, CL, QP, JD, and PL analyzed the data. HL provided expert guidance and reviewed the paper. All the authors gave the final approval of the manuscript.

\section{FUNDING}

This work was supported by the Guangxi Key Research and Development Program (GuiKe-17195005) and by the Health and Family Planning Commission of Guangxi Zhuang Autonomous Region (Z20180709).

\section{REFERENCES}

1. Sun X, Su S, Chen C, Han F, Zhao C, Xiao W, et al. Longterm outcomes of intensity-modulated radiotherapy for 868 patients with nasopharyngeal carcinoma: an analysis of survival and treatment toxicities. Radiother Oncol. (2014) 110:398-403. doi: 10.1016/j.radonc.2013. 10.020

2. Wu LR, Liu YT, Jiang N, Fan YX, Wen J, Huang SF, et al. Ten-year survival outcomes for patients with nasopharyngeal carcinoma receiving intensity-modulated radiotherapy: an analysis of 614 patients from a single center. Oral Oncol. (2017) 69:26-32. doi: 10.1016/j.oraloncology.2017. 03.015

3. Tang XR, Li YQ, Liang SB, Jiang W, Liu F, Ge WX, et al. Development and validation of a gene expression-based signature to predict distant metastasis in locoregionally advanced nasopharyngeal carcinoma: a retrospective, multicentre, cohort study. Lancet Oncol. (2018) 19:382-93. doi: 10.1016/S1470-2045(18)30 080-9

4. Chen L, Hu CS, Chen XZ, Hu GQ, Cheng ZB, Sun Y, et al. Concurrent chemoradiotherapy plus adjuvant chemotherapy versus concurrent chemoradiotherapy alone in patients with locoregionally advanced nasopharyngeal carcinoma: a phase 3 multicentre randomised controlled trial. Lancet Oncol. (2012) 13:163-71. doi: 10.1016/S1470-2045(11)7 0320-5

5. Liu MT, Chen MK, Huang CC, Huang CY. Prognostic value of molecular markers and implication for molecular targeted therapies in nasopharyngeal carcinoma: an update in an era of new targeted molecules development. World J Oncol. (2015) 6:243-61. doi: 10.14740/wjon610w

6. Li YF, Ding JW, Liao LM, Zhang ZL, Liao SS, Wu Y, et al. Expression of programmed death ligand-1 predicts poor outcome in nasopharyngeal carcinoma. Mol Clin Oncol. (2017) 7:378-82. doi: 10.3892/mco.201 7.1318

7. Parekh V, Jacobs MA. Radiomics: a new application from established techniques. Expert Rev Precis Med Drug Dev. (2016) 1:207-26. doi: 10.1080/23808993.2016.1164013

8. Li M, Zhang J, Dan Y, Yao Y, Dai W, Cai G, et al. A clinicalradiomics nomogram for the preoperative prediction of lymph node metastasis in colorectal cancer. J Transl Med. (2020) 18:46. doi: 10.1186/s12967-020-02215-0

9. Vaidya P, Bera K, Gupta A, Wang X, Corredor G, Fu P, et al. CT derived radiomic score for predicting the added benefit of adjuvant chemotherapy following surgery in atage I, II resectable non-small cell lung cancer: a retrospective multi-cohort study for outcome prediction. Lancet Digit Health. (2020) 2:e116-e28. doi: 10.1016/S2589-7500(20)30 002-9

10. Huang YQ, Liang CH, He L, Tian J, Liang CS, Chen X, et al. Development and validation of a radiomics nomogram for preoperative prediction of lymph node metastasis in colorectal cancer. J Clin Oncol. (2016) 34:215764. doi: 10.1200/JCO.2015.65.9128

11. Song J, Yin Y, Wang H, Chang Z, Liu Z, Cui L. A review of original articles published in the emerging field of radiomics. Eur J Radiol. (2020) 127:108991. doi: 10.1016/j.ejrad.2020.108991

12. Gillies RJ, Kinahan PE, Hricak H. Radiomics: Images are more than pictures, they are data. Radiol. (2016) 278:563-77. doi: 10.1148/radiol.20151 51169

13. Lambin P, Rios-Velazquez E, Leijenaar R, Carvalho S, van Stiphout RG, Granto P, et al. Radiomics: extracting more information from medical images using advanced feature analysis. Eur J Cancer. (2012) 48:4416. doi: 10.1016/j.ejca.2011.11.036

14. Zhang L, Fried DV, Fave XJ, Hunter LA, Yang J, Court LE. IBEX: an open infrastructure software platform to facilitate collaborative work in radiomics. Med Phys. (2015) 42:1341-53. doi: 10.1118/1.4908210

15. Gui J, Li H. Penalized Cox regression analysis in the high-dimensional and low-sample size settings, with applications to microarray gene expression data. Bioinformatics. (2005) 21:3001-8. doi: 10.1093/bioinformatics/ bti422

16. Hepp T, Schmid M, Gefeller O, Waldmann E, Mayr A. Approaches to regularized regression - A comparison between Gradient Boosting and the Lasso. Methods Inf Med. (2016) 55:422-30. doi: 10.3414/ME1601-0033

17. Vallières M, Kay-Rivest E, Perrin LJ, Liem X, Furstoss C, Aerts HJWL, et al. Radiomics strategies for risk assessment of tumour failure in head-and-neck cancer. Sci Rep. (2017) 7:10117. doi: 10.1038/s41598-017-10371-5

18. Zhang B, Tian J, Dong D, Gu D, Dong Y, Zhang L, et al. Radiomics features of multiparametric MRI as novel prognostic factors in advanced nasopharyngeal carcinoma. Clin Cancer Res. (2017) 23:4259-69. doi: 10.1158/1078-0432.CCR-16-2910

19. Zhang L, Zhou H, Gu D, Tian J, Zhang B, Dong D, et al. Radiomic nomogram: pretreatment evaluation of local recurrence in nasopharyngeal carcinoma based on MR imaging. J Cancer. (2019) 10:4217-25. doi: 10.7150/jca. 33345

20. Tang LQ, Li CF, Li J, Chen WH, Chen QY, Yuan LX, et al. Establishment and validation of prognostic nomograms for endemic nasopharyngeal carcinoma. J Natn Cancer Inst. (2015) 108:djv291. doi: 10.1093/jnci/ djv291

21. Sun XS, Liang YJ, Liu SL, Li XY, Chen QY, Guo SS, et al. Establishment and validation of a nomogram for predicting survival in patients with de novo metastatic nasopharyngeal carcinoma. Oral Oncol. (2019) 94:739. doi: 10.1016/j.oraloncology.2019.05.015

22. Song C, Yang S. A meta-analysis on the EBV DNA and VCA-IgA in diagnosis of nasopharyngeal carcinoma. Pak J Med Sci. (2013) 29:88590. doi: 10.12669/pjms.293.2907

23. Vo JH, Nei WL, Hu M, Phyo WM, Wang F, Fong KW, et al. Comparison of circulating tumour cells and circulating cell-free Epstein-Barr virus DNA in patients with nasopharyngeal carcinoma undergoing radiotherapy. Sci Rep. (2016) 6:13. doi: 10.1038/s41598-0160006-3

24. Li WF, Zhang Y, Huang XB, Du XJ, Tang LL, Chen L, et al. Prognostic value of plasma Epstein-Barr virus DNA level during posttreatment follow-up in the patients with nasopharyngeal carcinoma having undergone intensity-modulated radiotherapy. Chin J Cancer. (2017) 36:87. doi: 10.1186/s40880-017-0256-x

25. Huang XD, Zhou GQ, Lv JW, Zhou HQ, Zhang CW, Wu CF, et al. Competing risk nomograms for nasopharyngeal carcinoma in the 
intensity-modulated radiotherapy era: a big-data, intelligence platformbased analysis. Radiotherapy and oncology: J Eur Soc Ther Radiol Oncol. (2018) 129:389-95. doi: 10.1016/j.radonc.2018.09.004

26. Jiang F, Jin T, Feng XL, Jin QF, Chen XZ. Long-term outcomes and failure patterns of patients with nasopharyngeal carcinoma staged by magnetic resonance imaging in intensity-modulated radiotherapy era: The Zhejiang Cancer Hospital's experience. J Cancer Res Ther. (2015) 11(Suppl. 2):C17984. doi: 10.4103/0973-1482.168181

27. Balachandran VP, Gonen M, Smith JJ, Dematteo RP. Nomograms in oncology: more than meets the eye. Lancet Oncol. (2015) 16:e173e80. doi: 10.1016/S1470-2045(14)71116-7
Conflict of Interest: The authors declare that the research was conducted in the absence of any commercial or financial relationships that could be construed as a potential conflict of interest.

Copyright $\odot 2021$ Zhu, Huang, Liu, Chen, Jiang, Liao, Pang, Dang, Liu and Lu. This is an open-access article distributed under the terms of the Creative Commons Attribution License (CC BY). The use, distribution or reproduction in other forums is permitted, provided the original author(s) and the copyright owner(s) are credited and that the original publication in this journal is cited, in accordance with accepted academic practice. No use, distribution or reproduction is permitted which does not comply with these terms. 